\title{
Tumorous CD10 Is More Strongly Related to the Progression of Urothelial Carcinoma than Stromal CD10
}

\author{
ARISA KUMAGAI-TOGASHI, HIROSHI UOZAKI, YOSHINAO KIKUCHI, \\ SHIORI WATABE, SATOE NUMAKURA and MASATO WATANABE \\ Department of Pathology, Teikyo University School of Medicine, Tokyo, Japan
}

\begin{abstract}
Background/Aim: CD10 function in urothelial carcinoma (UC) remains controversial. We previously reported that miR-21 in UC may be a prognostic marker for cancer progression. The aim of this study was to examine the clinicopathological significance of CD10 expression in UC and its relationship with miR-21 expression. Materials and Methods: Immunohistochemistry for CD10 was performed on 232 UCs. CD10 expression in TCs and stroma was evaluated respectively, and its association with carcinogenesis and survival was analyzed. Results: High tumorous CD10 was significantly associated with higher tumor stage, histological grade and vessel infiltration, and poorer prognosis, whereas stromal CD10 was significantly associated with younger age, higher tumor stage, and vessel infiltration. On multivariable analysis, CD10 expression in TCs, miR-21 expression in TCs and TS, and tumor stage were independent prognostic factors. Conclusion: Tumorous CD10 is more strongly related to progression of UC than stromal CD10 and is an independent factor for UC prognosis.
\end{abstract}

Urothelial carcinoma (UC), which affects the upper (renal pelvis and ureters) and lower (bladder, urethra) urinary tract, is the sixth most common cancer type in males, with 330,400 new cases diagnosed and 123,100 deaths worldwide in 2012 (1). Most patients with superficial cancer prolong survival by transurethral resection of the bladder tumor (TUR-Bt). However, the prognosis of patients with advanced UC who underwent surgical treatment was not significantly improved. Therefore, further studies to elucidate the mechanism of urothelial carcinoma progression are needed.

Recently, interactions between tumor cells and the stroma surrounding tumor cells, as well as genetic and epigenetic

Correspondence to: Hiroshi Uozaki, Department of Pathology, Teikyo University School of Medicine, 2-11-1 Kaga, Itabashi-ku, Tokyo 1738605, Japan. Tel: +81 339641211, e-mail: uozaki@med.teikyo-u.ac.jp

Key Words: CD10, progression, survival, tumor stroma, urothelial carcinoma. alterations have been considered important for tumor progression. Cancer-associated fibroblasts (CAFs), a major component of the cancer stroma, contribute to tumor proliferation, invasion, metastasis, angiogenesis, and resistance to chemotherapy (2).

CD10 is a zinc-dependent cell surface neutral metallopeptidase that inactivates several bioactive peptides. CD10 is expressed in not only hematopoietic cells but also in nonhematopoietic tissues, including epithelial cells of the kidneys, intestine, liver, lungs, and prostate, endometrial stromal cells and breast myoepithelial cells (3). It was also reported that several neoplasms express CD10, whose function in cancer varies by tissue type. CD10 expression in tumor cells is a favorable prognostic factor in cervical carcinoma (4), but inversely indicates an unfavorable prognosis in several solid tumors such as gastric cancer (5), colorectal cancer (6), pancreatic endocrine tumor (7), and melanoma (8). CD10 expression in the tumor stroma of breast cancer (9), pancreatic cancer (10) and non-small cell lung carcinoma (11) was correlated with an unfavorable prognosis.

In UC, CD10 expression was found to be associated with tumor progression and an unfavorable prognosis, whereas Seiler et al. reported that high CD10 expression was a favorable prognostic factor in lymph node-positive bladder cancer patients (12). CD10 function in UC remains controversial.

We have previously reported that miR-21 may be an unfavorable prognostic marker in UC (13). In this study, we examined the clinicopathological significance of CD10 expression in both tumor cells and the stroma.

\section{Materials and Methods}

Samples from patients. We used the same samples as in our previous report (13). A brief summary of the samples and patients is shown in Table I. A total of $232 \mathrm{UC}$ samples were used, that were obtained from the archives of the Department of Pathology, Teikyo University Hospital from 2008 to 2012 . All the cases were first diagnoses of UC. One hundred and forty-eight samples were obtained from patients treated with transurethral resection of the bladder tumor and the other 84 samples were obtained from surgical resection. 
All tumors were staged according to the 8th TNM classification system of the Union for International Cancer Control (14). Histological grade was evaluated according to the World Health Organization/International Society of Urologic Pathology classification (15). The median follow-up period was 6.3 years (standard deviation, 0.31 years). Overall survival (OS) was defined as the period from surgery to death or last observation.

This study was approved by the Research Ethics Review Board of the Teikyo University School of Medicine (No. 15-067, 30 September 2015).

Tissue microarray construction. Tissue microarrays (TMA) were constructed using a manual tissue arrayer (Azumaya, Tokyo, Japan). Two 2.0-mm-diameter cores were obtained from representative tumor areas of formalin-fixed paraffin-embedded tissue blocks and arranged in TMA blocks. The TMA blocks were sliced at $3-\mu \mathrm{m}$ thickness and placed on slides. Suitable cases were defined as tumors occupying more than $50 \%$ of the core area.

Immunohistochemistry. Tissue sections were deparaffinized with xylene and rehydrated with graded ethanol. Antigen retrieval was performed by boiling at $98^{\circ} \mathrm{C}$ for $40 \mathrm{~min}$ in $0.01 \mathrm{~mol} / 1$ sodium citrate buffer ( $\mathrm{pH}$ 6.0). Endogenous peroxidase was quenched with $3 \% \mathrm{H}_{2} \mathrm{O}_{2}$ in distilled water for $5 \mathrm{~min}$. The primary antibody against CD10 (1:100, clone 56C6; Leica biosystems, Wetzlar, Germany) was applied for $30 \mathrm{~min}$ at room temperature. The sections were visualized by the HRP-labeled polymer method (EnVision FLEX systems, Dako, Denmark) according to the manufacturer's protocol and counterstained with hematoxylin. The sections were then dehydrated with graded ethanol, cleared with xylene, and coverslipped.

The expression level in the cell membrane and/or in the cytoplasm of tumor cells (TCs), and stroma surrounding tumor cells (TS) was semi-quantitatively evaluated according to the intensity (categorized as 0 [absent], 1 [weak], 2 [moderate], or 3 [strong]), and the percentage of positively stained TCs and TS (scored as 0 [0\%-5\% positive], 1 [6\%-25\%], 2 [26\%-50\%], 3 [51\%-100\%]). For the immunostaining score, the intensity and positivity scores were multiplied, resulting in a value between 0 and 9 . When the immunostaining score in TCs was higher than 3 and the immunostaining score in TS was higher than 1, it was classified as high expression. We also used the data from our previous study concerning miR-21 in UC (13).

Statistical analysis. The relationship between CD10 expression and clinicopathological features of patients was statistically evaluated by the chi-square test and Fisher's exact test. The correlation between CD10 expression and miR-21 expression was examined by the chi-square test. Survival was analyzed using the Kaplan-Meier method and curves were compared using the log-rank test. A Cox proportional hazard model was used in the univariate and multivariate analyses of survival. $p<0.05$ was considered significant.

\section{Results}

CD10 expression in urothelial carcinoma. Immunostaining of CD10 was observed in the cytoplasm and cell membrane of TCs and TS in UC (Figure 1). The results are summarized in Table II. High expression of CD10 in TC
Table I. Summary of the patients with urothelial carcinoma.

\begin{tabular}{|c|c|}
\hline Clinicopathological features & $\mathrm{N}$ \\
\hline \multicolumn{2}{|l|}{ Gender } \\
\hline Male & 186 \\
\hline Female & 46 \\
\hline Age, mean (range) & $69(29-92)$ \\
\hline \multicolumn{2}{|l|}{ Locus } \\
\hline Bladder & 187 \\
\hline Ureter & 23 \\
\hline Renal pelvis & 22 \\
\hline \multicolumn{2}{|l|}{ Tumor stage } \\
\hline pTa & 109 \\
\hline pT1 & 61 \\
\hline pT2 & 33 \\
\hline pT3 & 21 \\
\hline pT4 & 8 \\
\hline \multicolumn{2}{|l|}{ Histological grade } \\
\hline Low & 87 \\
\hline High & 145 \\
\hline \multicolumn{2}{|l|}{ Treatment } \\
\hline TUR-Bt & 148 \\
\hline Surgery & 84 \\
\hline \multicolumn{2}{|l|}{ Lymphatic infiltration** } \\
\hline Absent & 215 \\
\hline Present & 17 \\
\hline \multicolumn{2}{|l|}{ Vascular infiltration** } \\
\hline Absent & 215 \\
\hline Present & 17 \\
\hline \multicolumn{2}{|l|}{ Nodal involvement** } \\
\hline Absent & 222 \\
\hline Present & 10 \\
\hline
\end{tabular}

**Clinically negative specimens such as TUR-Bt specimens were classified as negative.

was found in 27 of $232(11.6 \%)$ cases, and CD10 expression in TS was found in 28 of $232(12.1 \%)$ cases. To evaluate heterogeneity of CD10 expression in TCs, five conventional formalin-fixed paraffin embedded (FFPE) sections were stained for CD10. Although heterogeneity of CD10 expression between the tumor center and the invasion front was not significant in any section, different immunostaining patterns, such as superficial cell-positive pattern, basal cell-positive pattern, and diffusely positive pattern, among the five cases were observed in the neoplastic epithelium.

Relationship between CD10 expression and clinicopathological features. The relationship between CD10 expression and clinicopathological features in UC patients is shown in Table III. High expression of CD10 in TCs was significantly associated with histological grade $(p=0.017)$, higher tumor stage $(p=0.004)$, the presence of lymphatic infiltration $(p=0.003)$, and the presence of vascular infiltration $(p<0.001)$. No significant correlation of CD10 

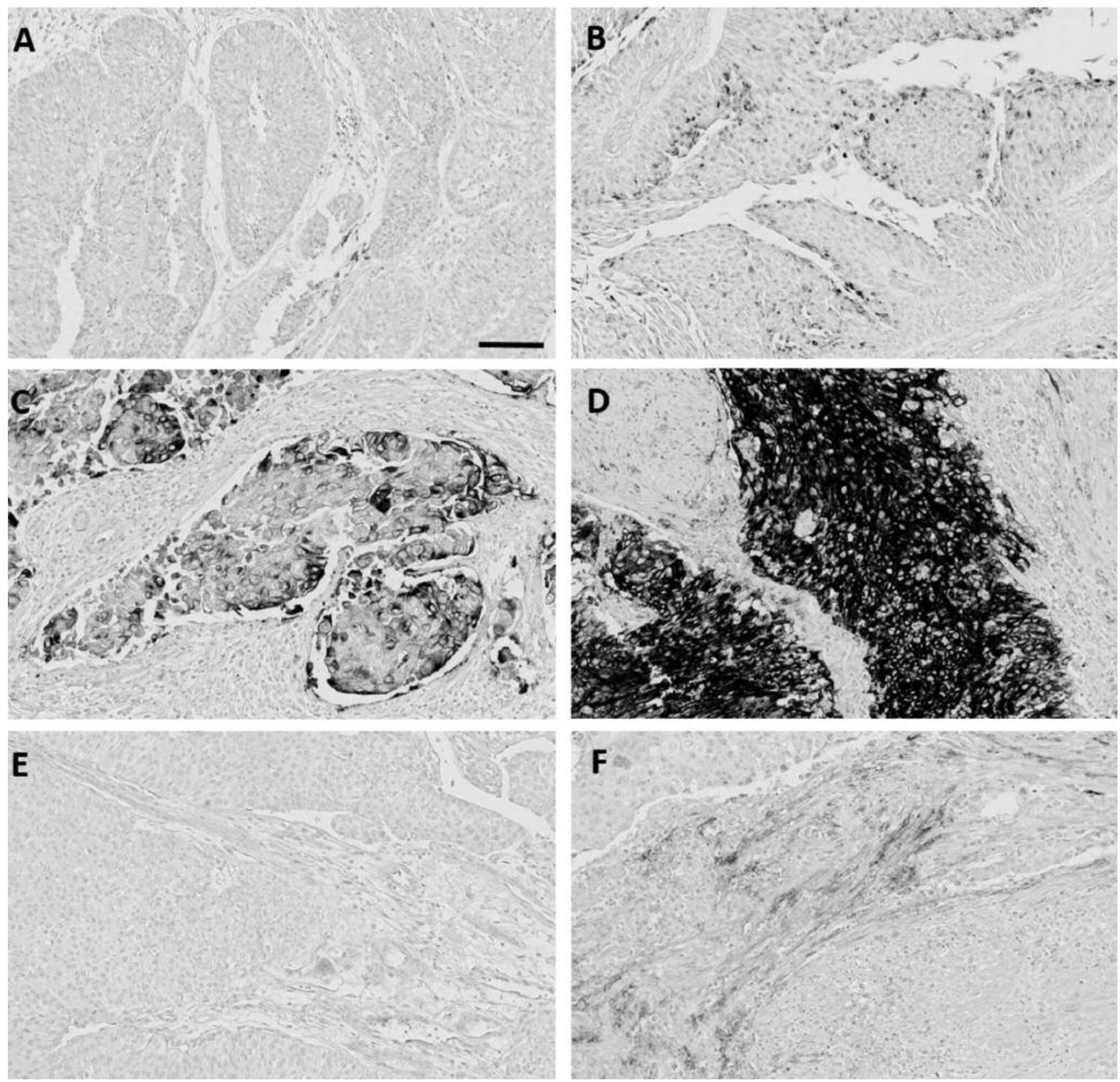

Figure 1. Representative image of CD10 expression in urothelial carcinoma. A-D: CD10 expression in tumor cells (TCS). E and F: CD10 expression in TS. (A) Immunostaining score is $0=$ intensity $0 \times$ positivity $0,(B)$ score is $2=1 \times 2,(C)$ score is $4=2 \times 2$, and (D) score $6=3 \times 2$ in TCs. (E) Immunostaining score is $0=0 \times 0$, and $(F)$ score is $3=3 \times 1$ in TS. Original magnification, $\times 200$. Scale bar $=100 \mu \mathrm{m}$.

expression in TCs with age, gender, tumor locus, or nodal involvement was found. The expression of CD10 in TS was significantly associated with younger age $(p=0.013)$, higher tumor stage $(p=0.005)$, the presence of lymphatic infiltration $(p<0.001)$, the presence of vascular infiltration $(p=0.008)$, and presence of nodal involvement $(p=0.023)$. No significant correlation of CD10 expression in TS with gender, histological grade, or tumor locus was found.
Relationship between CD10 expression and miR-21 expression. Twenty-one of $232(9.0 \%)$ cases were classified as high expression of miR-21 in TCs, and 15 of $232(6.5 \%)$ were classified as high expression of miR-21 in TS, in our previous report (13). To evaluate the correlation between CD10 expression and miR-21 expression in TCs and TS, we employed Chi-square tests. CD10 expression in TCs/TS was not correlated with the expression of miR-21. 
Table II. Distribution of immunostaining score.

\begin{tabular}{lcr}
\hline Score & Tumor cells & Stroma \\
\hline 0 & 139 & 204 \\
1 & 28 & 17 \\
2 & 38 & 9 \\
3 & 2 & 2 \\
4 & 11 & 0 \\
6 & 14 & 0 \\
9 & 0 & 0 \\
\hline
\end{tabular}

Relationship between CD10 expression and patient survival. Surgically resected cases were used for survival analysis. We used Kaplan-Meier survival curves to analyze the prognostic value of CD10 in UC. High CD10 expression in TCs was significantly correlated with unfavorable overall survival $(p=0.00277)$ (Figure 2). CD10 expression in TS was not of prognostic value.

To evaluate whether CD10 expression in TCs was an independent factor of overall survival for UC patients, a univariate Cox proportional hazard model was used to estimate the individual hazard ratio for all clinicopathological parameters. The results demonstrated that overall survival was significantly related to CD10 expression in TCs, miR-21 expression in TCs and TS, and tumor stage $(p<0.05)$. A multivariate analysis was performed with tumor stage (high/low), high CD10 expression in TCs, miR-21 expression in TCs and TS, histological grade, and tumor locus. CD10 expression in TCs, and miR-21 expression in TCs and TS were found to be independent prognostic factors $(p<0.05)$ (Table IV).

\section{Discussion}

CD10 is widely distributed in organs and plays a key role in the regulation of various biological functions (16). CD10 is present on the brush border of proximal tubules and on the glomerular epithelium (17); however, the function of CD10 in urinary organs is not clear. Although normal urothelial epithelium does not express CD10 (18), there are studies reporting a positive expression (19). Normal mucosa exhibited varying levels of CD10 expression in our trial (data not shown). Expression of CD10 was not different in the urothelial tract, renal pelvis, ureters, or urinary bladder in this study.

CD10 in UC was reported in several studies $(12,17-25)$. Most studies focused on CD10 expressed by tumor cells. High CD10 expression in TCs was related to higher tumor stage in most studies $(17,22-24)$ and high histological grade $(17,22-25)$. CD10 expression has been reported to be related to poor prognosis in $\mathrm{UC}(17,21)$, except for in one study

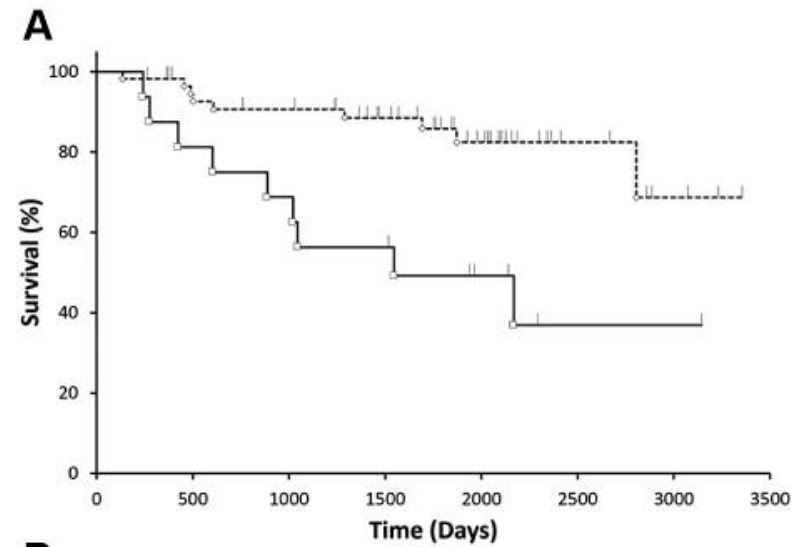

B

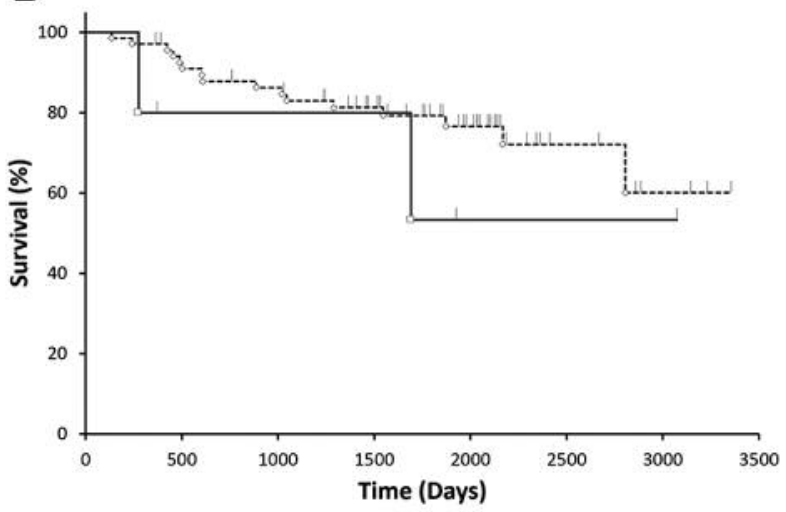

Figure 2. Overall survival curves by the Kaplan-Meier method for 84 patients with urothelial carcinoma according to high CD10 expression (solid lines) or low CD10 expression (dotted lines). (A) High CD10 expression in TCs was significantly associated with unfavorable overall survival ( $p=0.00277)$. (B) CD10 expression in TS was not related to survival.a

(12). In this study, CD10 expression in TCs was related to tumor stage, histological grade, vessel infiltration, and poor prognosis. CD10 was correlated with tumor progression of UC in our study. The proteolytic function of CD10 may contribute to these relationships.

On the other hand, stromal CD10 in UC is less studied than tumorous CD10. CD10 in TS is related to poor prognosis and higher tumor stage in colorectal cancer (26), pancreatic cancer $(10)$, breast cancer $(9,27)$, and non-small cell lung carcinoma (11). We examined CD10 in TS to clarify the difference in CD10 function between TCs and TS. The expression of CD10 in TS was weaker than that in TCs. The stromal CD10 was related to tumor stage, vessel infiltration, and nodal involvement, but not with overall survival. There is only one report regarding stromal CD10 (21), which also found no relationship between stromal CD10 and prognosis.

In our previous report we showed that stromal miR-21 had almost the same effects as tumorous miR-21 (13); however, 
Table III. Relationship between clinicopathological features and CD10 expression in tumor cells and stroma.

\begin{tabular}{|c|c|c|c|c|c|c|}
\hline & \multicolumn{2}{|c|}{ Tumor cells } & \multirow[b]{2}{*}{$p$-Value } & \multicolumn{2}{|c|}{ Stroma } & \multirow[b]{2}{*}{$p$-Value } \\
\hline & Low $(\mathrm{N}=205)$ & High $(\mathrm{N}=27)$ & & Negative $(N=204)$ & Positive $(\mathrm{N}=28)$ & \\
\hline \multicolumn{7}{|l|}{ Gender } \\
\hline Male & 165 & 21 & 0.940 & 163 & 23 & 1.000 \\
\hline Female & 40 & 6 & & 41 & 5 & \\
\hline \multicolumn{7}{|l|}{ Age (years) } \\
\hline mean \pm SD & $69.5 \pm 10.8$ & $68.5 \pm 8.53$ & 0.645 & $70.0 \pm 10.7$ & $64.8 \pm 8.4$ & $0.013 *$ \\
\hline \multicolumn{7}{|l|}{ Locus } \\
\hline Bladder & 166 & 21 & 0.923 & 161 & 26 & 0.217 \\
\hline Ureter & 19 & 3 & & 21 & 1 & \\
\hline Renal pelvis & 20 & 3 & & 22 & 1 & \\
\hline \multicolumn{7}{|l|}{ Tumor stage } \\
\hline pTa or pT1 & 157 & 13 & $0.004 *$ & 156 & 14 & $0.005 *$ \\
\hline pT2 or higher & 48 & 14 & & 48 & 4 & \\
\hline \multicolumn{7}{|l|}{ Histological grade } \\
\hline Low & 83 & 4 & $0.017 *$ & 76 & 11 & 1 \\
\hline High & 122 & 23 & & 128 & 17 & \\
\hline \multicolumn{7}{|c|}{ Lymphatic infiltration $* *$} \\
\hline Absent & 195 & 21 & $0.003 *$ & 195 & 21 & $<0.001 *$ \\
\hline Present & 10 & 6 & & 9 & 7 & \\
\hline \multicolumn{7}{|c|}{ Vascular infiltration $* *$} \\
\hline Absent & 195 & 20 & $<0.001^{*}$ & 193 & 22 & $0.008 *$ \\
\hline Present & 10 & 7 & & 11 & 6 & \\
\hline \multicolumn{7}{|c|}{ Nodal involvement $* *$} \\
\hline Absent & 196 & 26 & 1.000 & 198 & 24 & $0.023 *$ \\
\hline Present & 9 & 1 & & 6 & 4 & \\
\hline
\end{tabular}

SD: Standard deviation; *statistically significant, $p<0.05$; **clinically negative specimens such as TUR-Bt specimens are classified as negative.

Table IV. Prognostic factors for survival of patients on univariate and multivariate analyses.

\begin{tabular}{lcc}
\hline Characteristics & Unadjusted risk ratio $(95 \% \mathrm{CI} ; p$-Value) & Adjusted risk ratio $(95 \% \mathrm{CI} ; p$-Value) \\
\hline CD10 expression in TC & $4.05(1.60-10.2 ; 0.00308)$ & $4.67(1.62-13.5 ; 0.00444)$ \\
CD10 expression in TS & $1.91(0.435-8.38 ; 0.392)$ & $0.604(0.0706-5.16 ; 0.645)$ \\
miR-21 expression in TC & $3.79(1.32-10.9 ; 0.0136)$ & $4.88(1.35-17.6 ; 0.0155)$ \\
miR-21 expression in TS & $6.55(2.28-18.8 ; 0.000481)$ & $4.27(1.04-17.4 ; 0.0435)$ \\
Histological grade (Low) & $1.15(0.377-3.49 ; 0.808)$ & $2.15(0.611-7.56 ; 0.233)$ \\
Locus (renal pelvis) & $0.475(0.132-1.72 ; 0.256)$ & $0.559(0.136-2.30 ; 0.421)$ \\
Locus (ureter) & $0.573(0.182-1.80 ; 0.341)$ & $0.383(0.0999-1.47 ; 0.161)$ \\
Tumor stage (2 or higher) & $3.13(1.08-9.01 ; 0.0349)$ & $2.95(0.831-10.5 ; 0.0946)$ \\
\hline
\end{tabular}

95\%CI: $95 \%$ Confidence interval.

stromal CD10 was less related to tumor progression in UC. The proteolytic function of CD10 in tumor stroma may be weaker in UC than in other cancers.

CD10 is a cell surface zinc-dependent metallopeptidase that helps cancer cells invade the adjacent matrix (23). However, CD10 is widely distributed in several cells. Thus, as cells other than tumor cells may be influenced by antiCD10 therapy, specialized delivery systems must be developed for CD10-targeted therapy. In our study, CD10 and miR-21 were independent factors for UC prognosis, suggesting that both CD10 and miR-21 are potential therapeutic targets.

\section{Conflicts of Interest}

The Authors declare no conflicts of interest regarding this study. 


\section{Acknowledgements}

This work was supported by JSPS KAKENHI Grant Number 16K08658.

\section{References}

1 Torre LA, Bray F, Siegel RL, Ferlay J, Lortet-Tieulent J and Jemal A: Global cancer statistics, 2012. CA Cancer J Clin 65: 87-108, 2015.

2 Shiga K, Hara M, Nagasaki T, Sato T, Takahashi $H$ and Takeyama H: Cancer-associated fibroblasts: their characteristics and their roles in tumor growth. Cancers (Basel) 7: 2443-2458, 2015.

3 Chu P and Arber DA: Paraffin-section detection of CD10 in 505 nonhematopoietic neoplasms. Frequent expression in renal cell carcinoma and endometrial stromal sarcoma. Am J Clin Pathol 113: 374-382, 2000.

4 Terauchi M, Kajiyama H, Shibata K, Ino K, Mizutani S and Kikkawa F: Anti-progressive effect of neutral endopeptidase 24.11 (NEP/CD10) on cervical carcinoma in vitro and in vivo. Oncology 69: 52-62, 2005.

5 Tajima Y, Yamazaki K, Nishino N, Morohara K, Yamazaki T, Kaetsu T, Suzuki S, Kawamura M, Kumagai K and Kusano M: Gastric and intestinal phenotypic marker expression in gastric carcinomas and recurrence pattern after surgery-immunohistochemical analysis of 213 lesions. Br J Cancer 91: 13421348, 2004.

6 Fujita S, Taniguchi H, Yao T, Shimoda T, Ueno H, Hirai T and Ohue M: Multi-institutional study of risk factors of liver metastasis from colorectal cancer: correlation with CD10 expression. Int J Colorectal Dis 25: 681-686, 2010.

7 Deschamps L, Handra-Luca A, O'Toole D, Sauvanet A, Ruszniewski P, Belghiti J, Bedossa P and Couvelard A: CD10 expression in pancreatic endocrine tumors: correlation with prognostic factors and survival. Hum Pathol 37: 802-808, 2006.

8 Oba J, Nakahara T, Hayashida S, Kido M, Xie L, Takahara M, Uchi H, Miyazaki S, Abe T, Hagihara A, Moroi Y and Furue M: Expression of CD10 predicts tumor progression and unfavorable prognosis in malignant melanoma. J Am Acad Dermatol 65: 1152-1160, 2011.

9 Kim HS, Kim GY, Kim YW, Park YK, Song JY and Lim SJ: Stromal CD10 expression and relationship to the Ecadherin/beta-catenin complex in breast carcinoma. Histopathol 56: 708-719, 2010.

10 Ikenaga N, Ohuchida K, Mizumoto K, Cui L, Kayashima T, Morimatsu K, Moriyama T, Nakata K, Fujita H and Tanaka M: $\mathrm{CD} 10+$ pancreatic stellate cells enhance the progression of pancreatic cancer. Gastroenterology 139: 1041-1051, 2010.

11 Gurel D, Kargi A, Karaman I, Onen A and Unlu M: CD10 expression in epithelial and stromal cells of non-small cell lung carcinoma (NSCLC): a clinic and pathologic correlation. Pathol Oncol Res 18: 153-160, 2012.

12 Seiler R, von Gunten M, Thalmann GN and Fleischmann A: High CD10 expression predicts favorable outcome in surgically treated lymph node-positive bladder cancer patients. Hum Pathol 43: 269-275, 2012.
13 Ohno R, Uozaki H, Kikuchi Y, Kumagai A, Aso T, Watanabe M, Watabe S, Muto S and Yamaguchi R: Both cancerous miR-21 and stromal miR-21 in urothelial carcinoma are related to tumour progression. Histopathol 69: 993-999, 2016.

14 Brierley JD, Gospodarowicz MK and Wittekind C: TNM Classification of Malignant Tumours 8th ed, Hoboken, NJ, Wiley-Blackwell, 2017.

15 Epstein JI, Amin MB, Reuter VR and Mostofi FK: The World Health Organization/International Society of Urological Pathology consensus classification of urothelial (transitional cell) neoplasms of the urinary bladder. Bladder Consensus Conference Committee. Am J Surg Pathol 22: 1435-1448, 1998.

16 Maguer-Satta V, Besancon R and Bachelard-Cascales E: Concise review: neutral endopeptidase (CD10): a multifaceted environment actor in stem cells, physiological mechanisms, and cancer. Stem Cells 29: 389-396, 2011.

17 Langner C, Ratschek M, Rehak P, Schips L and Zigeuner R: $\mathrm{CD} 10$ is a diagnostic and prognostic marker in renal malignancies. Histopathol 45: 460-467, 2004.

$18 \mathrm{Kim}$ MK and Kim S: Immunohistochemical profile of common epithelial neoplasms arising in the kidney. Appl Immunohistochem Mol Morphol 10: 332-338, 2002.

19 Murali R and Delprado W: CD10 immunohistochemical staining in urothelial neoplasms. Am J Clin Pathol 124: 371-379, 2005.

20 Bircan S, Candir O, Kapucuoglu N, Serel TA, Ciris M and Karahan N: CD10 expression in urothelial bladder carcinomas: a pilot study. Urol Int 77: 107-113, 2006.

21 Abdou AG: CD10 expression in tumour and stromal cells of bladder carcinoma: an association with bilharziasis. APMIS 115: 1206-1218, 2007.

22 Bahadir B, Behzatoglu K, Bektas S, Bozkurt ER and Ozdamar SO: CD10 expression in urothelial carcinoma of the bladder. Diagn Pathol 4: 38, 2009.

23 Jang TJ: CD10 Is Again Expressed at a Certain Stage during the Neoplastic Process of Bladder Transitional Cell Carcinomas. Cancer Res Treat 44: 262-266, 2012.

24 Omran OM: CD10 and E-cad expression in urinary bladder urothelial and squamous cell carcinoma. J Environ Pathol Toxicol Oncol 31: 203-212, 2012.

25 Mohammed AS, Ali HH, Qasim BJ and Chaloob MK: CD10 and CA19.9 immunohistochemical expression in transitional cell carcinoma of the urinary bladder. Urol Ann 5: 81-85, 2013.

26 Ogawa H, Iwaya K, Izumi M, Kuroda M, Serizawa H, Koyanagi $\mathrm{Y}$ and Mukai K: Expression of CD10 by stromal cells during colorectal tumor development. Hum Pathol 33: 806-811, 2002.

27 Vo TN, Mekata E, Umeda T, Abe H, Kawai Y, Mori T, Kubota Y, Shiomi H, Naka S, Shimizu T, Murata S, Yamamoto H, Ishida $\mathrm{M}$ and Tani T: Prognostic impact of CD10 expression in clinical outcome of invasive breast carcinoma. Breast Cancer 22: 117128, 2015. 